\title{
Animals on the menu
}

\section{W} strange relationship we humans and we cuddle them. We feed them, we play with them, we make money from them, we hunt them, we build tourism around them, we breed them, we teach them tricks and we pass legislation to protect them. In short, we use them for any purpose we wish and, generally, we realise that with this power comes some responsibility.

With the exception of those that are regarded as pests, our reaction towards animals is usually positive. But occasionally, humans do deviate and torture animals, which debases our human dignity and such mistreatment is rightly seen as an abomination. Feeding and caring for animals such that they end up sizzling in our frying pans, however, is seen as another matter. Creating inhumane environments for high-throughput chicken production is generally accepted, apparently because it has reduced the price of succulent chicken breasts to a level where they are a commodity rather than an occasional luxury. Society has obviously accepted the concept of 'man the hunter' - even when it equates today to 'man the shopper'and has balanced animal rights against practical benefits to our agricultural sector.

But there is an increasing uneasiness in some parts of the population about the use of animals in scientific research. For years now, scientists have been the target of various animal rights groups. The word 'target' is unfortunately apt as some extreme animal lovers feel that violence against equipment or even scientists is acceptable when they believe that animals are being tortured in research laboratories. Fortunately, these extremists are few in number, but other, less radical groups also question whether scientists can be trusted in their use of animals and press for legislative intervention. And they are beginning to see results. The German government has recently included animals in a pledge to protect them for future generations-which is seen as a possible entry point for future challenges to accepted laboratory practices.
In the USA, politicians, scientists and animal welfare groups are debating whether rodents and birds should be excluded from laws that tighten restrictions on animal-based research. And any experiment involving a primate can now only be described as an extremely delicate ethical exercise that raises almost the same concerns as would the same treatment of a human. So, can we scientists really be trusted in the way we treat animals? Do we really need to do these kinds of experiments? Should we have to hide from the animal-welfare terrorists? Should annual reports need to disguise the fact that the institute has a large animal facility for research purposes?

Of course my response carries the bias of a scientist. So let's look at it a different way. What would happen if there were no experiments with animals in the laboratory? We could, of course, replace them with a 'cell only' regime and this has indeed happened for many. But there are problems with these alternatives: the cells we grow are not 'normal' and live in an alien environment. They are little more than a crude approximation of their predecessors that were recovered from a solid, tightly packed tissue, where they were a long way from fetal calf serum and very close to the paracrine influences of neighbouring cells. We could pursue many of our studies using cellular models, but after a while we would urgently need reality checks. Moreover, we would not be able to study certain biological events as many cells simply refuse to grow when they are away from 'home'.

In a world where animal rights have precedence over all other needs, we would also have no antibodies. This would not only hinder further research but also the development of useful diagnostics including food safety tests, pregnancy tests, monitoring of cancer treatments, etc. New drugs would be developed but at a slower rate if the hands of the researchers were tied too tightly. And they could not be tested in the same way as is currently required by the monitoring authorities. True, there are some non-animal screens, but animal tests often provide the first indications of safety problems with a new treatment. These are not infallible, but every year the development of some promising drug is abandoned during preclinical tests when such animal tests raise serious doubts about safety. A further loss would be all the studies on transgenic/knock out/knock in/conditional and otherwise mice. The impact of this technology on biological research has been truly phenomenal; it follows that we would dramatically lose out if such studies were precluded.

I suspect that the real target of animal rights activists are those studies where live animals are 'challenged' with experimental insults and, for example, neurological responses are monitored. But the Boris Karloff image often attributed to the scientist in such scenarios damages the work of many of their colleagues, even those who do not even get close to animals. Such experiments, I am assured, are essential and irreplacable. Presuming that this is so, then absolute transparency and rigid application of regulations aimed at minimising pain and stress to the animals are required. The funding bodies should be as vigilant as they would be for human trials and those deviating from the rules, as well as the people in charge of the institute, should be held legally responsible for any crimes or wrongdoings. The same should be true for other, less emotionally challenging, experiments that use animals. But this is quite different from stopping legitimate animal experiments that have been accepted by a monitoring body.

We, and here I mean the scientist and the non-scientist, need to be able to use animals in a proper manner. To block experiments with animals would be tantamount to blocking research. Surely this is not the goal of the majority of animal welfare groups who actively push for animal rights as a higher need than biomedical research: or is it?

\section{Frank Gannon}

DOI: 10.1093/embo-reports/kvf143 